\title{
A STABILITY THEOREM FOR MINIMUM EDGE GRAPHS WITH GIVEN ABSTRACT AUTOMORPHISM GROUP
}

\author{
BY \\ DONALD J. MCCARTHY (1) AND LOUIS V. QUINTAS
}

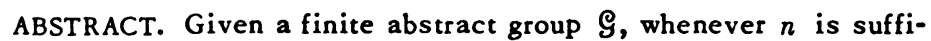
ciently large there exist graphs with $n$ vertices and automorphism group isomorphic to $\varrho$. Let $e(\mathcal{G}, n)$ denote the minimum number of edges possible in such a graph. It is shown that for each $\mathcal{G}$ there always exists a graph $M$ such that for $n$ sufficiently large, $e(\varrho, n)$ is attained by adding to $M$ a standard maximal component asymmetric forest. A characterization of the graph $M$ is given, a formula for $e(\varrho, n)$ is obtained (for large $n$ ), and the minimum edge problem is re-examined in the light of these results.
\end{abstract}

1. Introduction. Throughout this paper, automorphism groups of graphs will be regarded as abstract groups rather than permutation groups. It is well known [1] that given any finite group $\mathfrak{G}$, there always exists a graph whose automorphism group is isomorphic to $\mathcal{G}$. It is natural to consider the following problem: Given a finite group $\mathcal{G}$, for each positive integer $n$ decide whether or not there exists a graph on $n$ vertices having automorphism group isomorphic to $\mathcal{G}$, and if there do exist such graphs determine the minimum number $e(\mathcal{G}, n)$ of edges possible. For a survey of results leading to this and related problems see [4].

To date, this minimum edge problem has been completely solved only when $\mathcal{G}$ is the identity group, a symmetric group, a dihedral group, or the cyclic group of order 3 [13], [14], [5], [3]. See also [16]. An examination of these cases reveals the following pattern. For small values of $n$, the behavior of $e(\varrho, n)$ may be somewhat erratic, due to the fact that the graphs for which $e(\varrho, n)$ is attained may fluctuate wildly, and for sporadic values of $n$ no such graphs may exist. But eventually the situation becomes more stable: for $n$ sufficiently large $e(\varrho, n)$ is always defined and, indeed, is

Presented to the Society, August 30, 1972 under the title $A$ stability theorem for minimum edge graphs with given abstract group; received by the editors April $17,1973$.

AMS (MOS) subject classifications (1970). Primary 05C25, 20B25; Secondary 05C35, 05C05, 20D99.

Key words and phrases. Automorphism groups of graphs, minimum edge problem.

(1) Work of the first author was supported by National Science Foundation Grant GP.31723. 
attained by adding to some fixed graph $M$ a certain standard asymmetric forest. The point of the present paper is to show that this stability phenomenon occurs in general, for an arbitrary group $\mathcal{G}$. A precise statement of this result is given at the end of the next section.

The theorem obtained characterizes $M$ as a semireduced 9 -graph having minimum defect $d_{0}$ (see $\$ 2$ for definitions) and a minimum number $v_{0}$ of vertices. It establishes, for large $n$, the formula $e(\varrho, n)=n+d_{0}-c$, where $c$ denotes the number of components in a certain standard asymmetric forest on $n-v_{0}$ vertices. The basic properties of these forests are given in $\S 3$, and the proof of the stability theorem is given in $\$ 4$.

Some consequences of the theorem are examined in $\$ 5$; the minimum edge problem is reformulated, and observations are made regarding the stability graph $M$. These observations are applied, in $\$ 6$, to the case where $\&$ is commutative, and the cyclic case is treated in detail.

2. Preliminaries and notation. The bulk of the graph-theoretic terminology employed is in rough conformity with common usage [7], [11]. Throughout, all graphs are finite and undirected, without loops or multiple edges. Note that we permit the empty graph $\varnothing$, which has no vertices or edges.

For any graph $G$ let. $v(G), e(G), c(G)$ denote, respectively, the number of vertices, edges and connected components of $G$. By convention, $\varnothing$ is not a component of $G$. The cycle rank of $G$ will be denoted by $r(G)$ and is given by $r=e-v+c$. We introduce the defect $d(G)$ defined by $d=$ $r-c$.

An automorphism of $G$ is a permutation of the set of vertices of $G$ which preserves adjacency. The collection of all automorphisms of $G$ forms a group which will be denoted by $\mathcal{C}_{u t}(G)$. The notation $C_{u t}(G) \cong \varrho$ will mean that ( $u t(G)$ is isomorphic (as an abstract group) to the group $\varrho_{\text {; }}$ in this case we refer to $G$ as a 9 -graph. The identity group with only one element will sometimes be denoted by id, and an id-graph is termed asymmetric. By convention, $\varnothing$ is asymmetric.

Throughout, all groups are finite. The symmetric group of order $m$ ! is designated by $\mathcal{S}_{m}$, and $\mathcal{S}_{m}[\varrho]$ denotes the wreath product of $\varrho_{\text {by }} \mathcal{S}_{m}$. This last group is defined concretely in [12] and in $[9, \S 3]$, and an abstract description is given below. Begin by taking the direct product of $m$ copies of $\mathcal{G}_{\text {; say }} \mathfrak{T}=\varrho_{1} \times \mathscr{S}_{2} \times \ldots \times \mathscr{S}_{m}$ where $\mathscr{S}_{i} \cong \varrho_{\text {. Let }} \mathcal{S}_{m}$ act on $\mathfrak{I}$ by permuting the subscripts in the natural manner. Finally, $\delta_{m}[\mathcal{S}]$ is the semidirect product of $I$ by $\delta_{m}$ using this action. For details see [10, Chapter 6$]$ or $[9, \S 2]$. 
Wreath products arise naturally as automorphism groups of graphs all of whose components are isomophic [2]. In general, suppose $G=m_{1} G_{1}+m_{2} G_{2}+$ $\ldots+m_{k} G_{k}$ where the $G_{i}$ are connected, $G_{i}$ is not isomorphic to $G_{j}$ for $i \neq j$, the symbol + denotes disjoint union, and $m_{i} G_{i}$ is the disjoint union of $m_{i}$ copies of $G_{i}$. If $\mathscr{C}_{u t}\left(G_{i}\right) \cong \varrho_{i}$ then $\mathscr{C}_{u t}(G) \cong \mathcal{S}_{m_{1}}\left[\varrho_{1}\right] \times \delta_{m_{2}}\left[\varrho_{2}\right] \times \ldots$ $\times \delta_{m_{k}}\left[\varrho_{k}\right]$.

If $C$ is a connected nonempty graph, the multiplicity of $C$ in a graph $G$ is defined as the number of components of $G$ which are isomorphic to $C$. It follows from the preceding remarks that in a nonempty asymmetric graph all components have multiplicity 1. A graph $G$ is said to be reduced if $G$ has no proper subgraph $H$ which is a union of components of $G$ such that $\operatorname{Cut}(H) \cong$ Cut $(G)$. Equivalently, $G$ is reduced if and only if $G$ has no asymmetric components of multiplicity 1 . Observe that the empty graph is the only reduced asymmetric graph. It is clear that every graph $G$ can be decomposed in a unique manner as $R+A$ where $R$ is reduced, $A$ is asymmetric and $\mathscr{C l u t}_{u t}(G) \cong$ $\mathbb{Q u t}_{\mathrm{u}}(R)$.

Let $a(G)$ denote the number of nonisomorphic asymmetric components of $G$, and let $a_{1}(G)$ be the number of nonisomorphic asymmetric components of multiplicity $>1$ in $G$. Observe that if $C$ is an asymmetric component of multiplicity $m$ in $G$, then $C_{u t}(G)$ has a direct factor isomorphic to $\mathcal{S}_{m}[i d] \cong$ $\delta_{m}$. Using the uniqueness of the direct product decomposition of a finite group into indecomposable factors $\left[6\right.$, p. 130] and the indecomposability of $\mathcal{S}_{m}$, it follows that $a_{1}(G)$ cannot exceed the number of nontrivial symmetric groups which appear as direct factors in the decomposition of $C_{u t}(G)$, and the multiplicities $m_{i}$ of these asymmetric components must equal the degrees of the corresponding symmetric groups $\mathcal{S}_{m_{i}}$.

Let $s(\biguplus)$ denote the number of nontrivial direct factors in the standard decomposition of $\mathfrak{S}$ (into directly indecomposable groups) which are isomorphic to symmetric groups. We have just remarked that $a_{1}(G) \leq s\left(G_{)}\right.$for every $\oint_{\text {- }}$

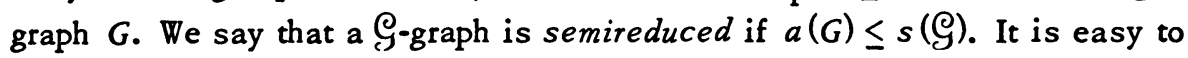

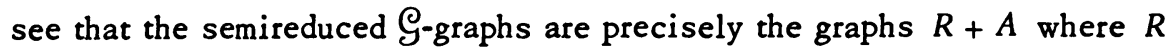
is a reduced 9 -graph and $A$ is a graph having at most $s(\mathcal{G})-a(R)$ components, each of which is asymmetric and of multiplicity 1 in $A$ and not isomorphic to any component of $R$. In particular, every reduced $\mathfrak{S}_{\text {-graph }}$ is semireduced.

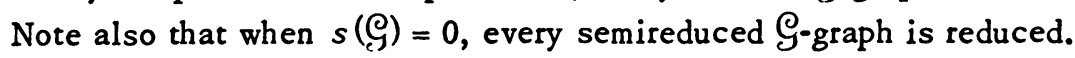

We are now prepared to state our main result.

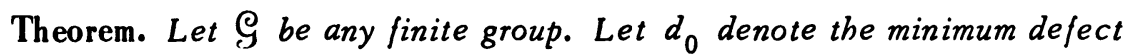
$d(S)$, where $S$ ranges over all semireduced G-graphs; among all such graphs 
which satisfy $d(S)=d_{0}$ let $M$ be one having the smallest number of vertices $v_{0}$.

Then for $n$ sufficiently large, $e(\varrho, n)=n+d_{0}-c\left(Q_{m, s}\right)$ where $m=$

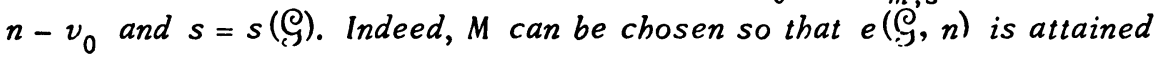
by $M+Q_{m, s}$

In the above, the graph $Q_{m, s}$ is a certain standard asymmetric forest. It has $m$ vertices and a maximum number of components none of which is isomorphic to any member of a fixed set of $s$ trees. The graphs $Q_{m, s}$ are defined precisely and their properties are investigated in the following section.

3. Standard asymmetric forests. Throughout we shall assume that all nonisomorphic asymmetric trees have been enumerated in some fixed order, $T_{1}, T_{2}, T_{3}, \ldots$, subject only to the condition that the trees are listed in order of increasing number of vertices, i.e. $v\left(T_{i}\right) \leq v\left(T_{i+1}\right)$.

Following Quintas [13], we define a graph $Q_{n}$ having $n$ vertices, obtained essentially by taking an initial segment of this standard list of asymmetric trees. More precisely, suppose $c$ is maximal so that $v\left(T_{1}+T_{2}+\ldots+T_{c}\right)$ $\leq n$. If equality holds here, take $Q_{n}=T_{1}+T_{2}+\ldots+T_{c}$; otherwise we modify this slightly, replacing $T_{c}$ by the first tree $T_{k}$ in the list having exactly $n-v\left(T_{1}+T_{2}+\ldots+T_{c-1}\right)$ vertices. Clearly $Q_{n}$ is asymmetric, and $c=c\left(Q_{n}\right)$ is the maximum number of components in any asymmetric forest on $n$ vertices. Since asymmetric trees on $n$ vertices exist except when $1<n<$ 7 , we see that $Q_{n}$ is defined for $n=1$ and $n \geq 7$.

We now make a slight generalization of this construction. In what follows, F will denote a finite collection of nonisomorphic asymmetric trees. The graph $Q_{n}(\mathfrak{T})$ is defined by precisely the same procedure as above, but after first deleting the elements of $\mathfrak{T}$ from our standard list of asymmetric trees. When $\mathfrak{T}=\varnothing$ we have $Q_{n}(\mathfrak{T})=Q_{n}$, and letting $\mathfrak{I}_{s}=\left\{T_{1}, T_{2}, \ldots, T_{s}\right\}$ define $Q_{n, s}=Q_{n}\left(\mathfrak{S}_{s}\right)$. A graph $G$ is said to be $\mathfrak{T}$-free if each element of $\mathfrak{T}$ has multiplicity zero in $G$.

Lemma 1. If $A$ is an asymmetric $\mathfrak{T}$-free graph then $d(A) \geq d\left(Q_{n}(\mathfrak{T})\right)$ uhenever $Q_{n}(\mathfrak{T})$ is defined and $n \geq v(A)$.

Proof. If $C$ is a component of $A$ then $d(C)=-1$ if $C$ is a tree, and $d(C) \geq 0$ otherwise. Thus if $c_{0}$ denotes the number of components of $A$ which are trees, we have $d(A) \geq-c_{0}$. Each of the $c_{0}$ trees which occur as components of $A$ is isomorphic to exactly one term in our standard list of asymmetric trees and none lies in $\mathfrak{T}$. It follows that the total number of vertices in the sum of the first $c_{0}$ trees in the list obtained by deleting $\mathfrak{T}$ from the standard list is at most $v(A)$. Since $n \geq v(A)$, it is clear from the construction 
that $Q_{n}(\mathfrak{T})$ has at least $c_{0}$ components. Thus $d\left(Q_{n}(\mathfrak{T})\right)=-c\left(Q_{n}(\mathfrak{T})\right) \leq$ $-c_{0} \leq d(A)$.

Since $e=v+d$ in general, it follows from the above result that $Q_{n}(\mathfrak{T})$ is a minimum edge graph among all asymmetric $\mathfrak{T}$-free graphs on $n$ vertices. When $\mathfrak{T}=\varnothing$ this yields $e(i d, n)=e\left(Q_{n}\right)$ whenever $Q_{n}$ is defined, as shown by Quintas [13].

Lemma 2. Given $\mathfrak{T}$ and a fixed integer $k \geq 0$, we have

$$
-1 \leq d\left(Q_{n+k}(\mathfrak{T})\right)-d\left(Q_{n}(\mathfrak{T})\right) \leq 0
$$

provided only that $n$ is sufficiently large. More precisely, there exists an integer $N_{k}(\mathfrak{T})$ so that whenever $n \geq N_{k}(\mathfrak{T}), Q_{n}(\mathfrak{T})$ exists and the above inequalities hold.

Proof. Assume for the moment that $Q_{n}(\mathfrak{T})$ exists whenever $n$ is sufficiently large. Let $x=d\left(Q_{n+k}(\mathfrak{T})\right)-d\left(Q_{n}(\mathfrak{T})\right.$ ). It is clear (e.g. from Lemma 1) that $x \leq 0$ whenever both $Q_{n+k}(\mathfrak{T})$ and $Q_{n}(\mathfrak{I})$ are defined. Observe that $-x$ is the number of components by which $Q_{n+k}(\mathfrak{I})$ exceeds $Q_{n}(\mathfrak{T})$. Thus if $n$ is so large that $Q_{n}(\mathfrak{T})$ already includes all available trees on at most $k$ vertices, then clearly $-x \leq 1$ as desired.

To complete the proof we need only obtain an integer $N_{0}(\mathfrak{T})$ such that $Q_{n}(\mathfrak{T})$ exists whenever $n \geq N_{0}(\mathfrak{T})$. Then $N_{k}(\mathfrak{I})$ can be taken as the larger of $N_{0}(\mathfrak{T})$ and $v\left(F_{k}(\mathfrak{I})\right)$, where $F_{k}(\mathfrak{I})$ is the sum of all nonisomorphic asymmetric $\mathfrak{T}$-free trees on at most $k$ vertices. Suppose $m$ is an integer such that whenever $n \geq m$ there exists a $\mathfrak{T}$-free tree on $n$ vertices. Letting $F=$ $F_{m-1}(\mathfrak{T})$ it is clear that we can take $N_{0}(\mathfrak{T})=m+v(F)$. Finally, we observe that $m$ can be any integer $\geq 7$ which exceeds the maximum number of vertices of any tree in $\mathfrak{T}$. For this last, we need only note that there exists an asymmetric tree on $n$ vertices whenever $n \geq 7$.

In what follows, we let $N_{k, s}=N_{k}\left(\mathfrak{T}_{s}\right)$. It will also be important in the sequel to obtain an integer $N_{0}(t)$ such that $Q_{n}(\mathfrak{T})$ exists for $n \geq N_{0}(t)$ whenever $\mathfrak{T}$ contains at most $t$ trees. To do this, assume $t>0$ and suppose $m_{t}$ is an integer such that the number of nonisomorphic asymmetric trees on $m_{t}$ vertices exceeds $t$. Clearly $n \geq m_{t}$ guarantees the existence of a $\mathfrak{T}$-free tree on $n$ vertices for all collections $\mathfrak{I}$ containing at most $t$ trees. Thus if $F$ denotes the sum of all nonisomorphic asymmetric trees having fewer than $m_{t}$ vertices, we can take $N_{0}(t)=m_{t}+v(F)$.

The existence of $m_{t}$ follows from the well-known fact that $a_{n}$, the number of nonisomorphic asymmetric trees on $n$ vertices, grows arbitrarily large with $n$. (This can be seen as follows. For $n \geq 7$, start with a path of length $n-2$, with vertices labelled consecutively as $v_{1}, v_{2}, \ldots, v_{n-1}$. At vertex $v_{i}$ 
append a path of length 1 to obtain a tree $G_{i}$ having $n$ vertices. For $2<$ $i \leq(n-1) / 2$ the $G_{i}$ are asymmetric and mutually nonisomorphic. Thus $a_{n} \geq$ $[(n-1) / 2]-2=[(n-5) / 2]$. Better information on $a_{n}$ can be obtained from [81.)

4. Proof of the stability theorem. We begin by observing that for any group $\mathfrak{G}$, there exist $\mathcal{G}$-graphs on $n$ vertices whenever $n$ is sufficiently large. For suppose $G_{0}$ is any $\mathcal{G}_{\text {-graph; }}$ existence of $G_{0}$ is guaranteed by [1]. Let $\mathfrak{T}$ denote the set of nonisomorphic asymmetric trees having multiplicity $>0$ in

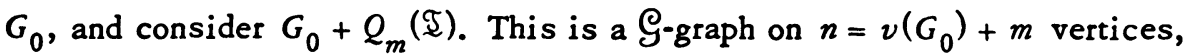
existing whenever $n \geq v\left(G_{0}\right)+N_{0}(\mathfrak{T})$.

We wish to show that for $n$ sufficiently large, the minimum number of edges for all $\mathcal{G}$-graphs on $n$ vertices is given by

$$
e_{n}=n+d_{0}-c\left(Q_{m, s}\right)
$$

and that $e_{n}$ is attained by a graph of the form $M+Q_{m, s}$ as indicated in the statement of the theorem. We prove the latter part first.

Thus, suppose $M$ is a semireduced $\oint_{-g r a p h}$ attaining minimum defect $d_{0}$ and having minimum number of vertices $v_{0}$. Letting $s=s(\zeta)$ as defined in $\$ 2$, consider the graph $M+Q_{m, s}$ on $n=v_{0}+m$ vertices. This graph

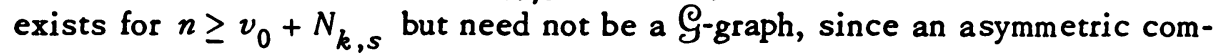
ponent of $M$ may be repeated in $Q_{m, s}$. In that case, however, in view of the minimality of $v_{0}$ any such component must have the same number of vertices as some element of $\mathfrak{T}_{s}$ not appearing in $M$. It is possible to replace all occurrences of such components in $M$ by appropriate elements of $\mathfrak{T}_{s}$ to obtain a semireduced $\varrho_{\text {-graph }} M_{1}$ whose asymmetric components all come from $\mathfrak{T}_{s}$. We have $d\left(M_{1}\right)=d_{0}, v\left(M_{1}\right)=v_{0}$ and $G_{n}=M_{1}+Q_{m, s}$ is a -graph on $n$ vertices with $e\left(G_{n}\right)=n+d\left(G_{n}\right)=e_{n}$ as desired.

To complete the proof of the theorem, suppose $G$ is any $\varrho$-graph on $n$ vertices; we will show that $e(G) \geq e_{n}$ provided only that $n$ is sufficiently large. Again let $s=s(\mathcal{G}), m=n-v_{0}$ and write $G=R+A$ where $R$ is a reduced $\varrho$-graph and $A$ is asymmetric. Decompose $A$ as $A_{s}+A_{*}$ where $A_{s}$ is the sum of all those components of $A$ isomorphic to elements of $\mathfrak{T}_{s}$. Let $\mathfrak{I}=\mathfrak{I}_{S} \cup \mathfrak{T}(R)$, where $\mathfrak{T}(R)$ denotes the set of all asymmetric trees having multiplicity $>0$ in $R$. Thus $G=\left(R+A_{s}\right)+A_{*}$ and $A_{*}$ is $\mathfrak{T}$-free and asymmetric. We distinguish two cases, according as $R+A_{s}$ is semireduced or not.

Case 1. $R+A_{s}$ is semireduced. Observe that $e(G)-e_{n}=x+y$ where $x=d\left(R+A_{s}\right)-d_{0}$ and $y=d\left(A_{*}\right)-d\left(Q_{m, s}\right)$. In the present case we have $x \geq 0$, and by Lemma $1, y \geq 0$ provided $v_{1} \geq v_{0}$, where $v_{1}=v\left(R+A_{s}\right)$. Thus 
assume $v_{1}<v_{0}$. By the minimality of $v_{0}$ we have $x>0$, hence need only show $y \geq-1$. Let $k=v_{0}-v_{1}$ so that $v\left(A_{*}\right)=m+k$. Since $k>0, Q_{m+k, s}$ is defined whenever $n$ (and hence $m$ ) is sufficiently large, and since $A_{*}$ is I-free we have $d\left(A_{*}\right) \geq d\left(Q_{m+k, s}\right)$. Thus $y \geq d\left(Q_{m+k, s}\right)-d\left(Q_{m, s}\right)$ and the desired result follows from I.emma 2 . More precisely, we obtain $y \geq-1$ whenever $n \geq v_{0}+N_{k, s}$ where $k=v_{0}-v_{1} \leq v_{0}$. So in any event, $x+y \geq 0$ whenever $n \geq v_{0}+N_{v_{0}, s^{\circ}}$

Case 2. $R+A_{s}$ is not semireduced. If we let $a\left(R+A_{s}\right)=s+t$ then $0<t \leq s$ and it is clear that $A_{s}$ has at least $t$ components and that $R$ has at least $t$ nonisomorphic asymmetric components not lying in $\mathfrak{T}_{s}$. Let $C_{1}$, $C_{2}, \ldots, C_{t}$ be such a set of components of $R$, and let $D_{1}, D_{2}, \ldots, D_{t}$ be components of $A_{s}$. Let $m_{i}$ be the multiplicity of $C_{i}$ in $R$ and, finally, let $H$ be the graph obtained from $R+A_{s}$ by replacing $m_{i}-1$ copies of $C_{i}$ by copies of $D_{i}$. Thus if we let $C=\Sigma\left(m_{i}-1\right) C_{i}$ and $D=\Sigma\left(m_{i}-1\right) D_{i}$, we have $H=R+A_{s}-C+D$.

Observe that if $Q$ is any asymmetric $\mathfrak{T}$-free graph and $G^{\prime}=H+Q$, then $G^{\prime}$ is a $\mathcal{G}_{\text {-graph }}$ and in the decomposition $G^{\prime}=R^{\prime}+A_{s}^{\prime}+A_{*}^{\prime}$, the $C_{i}$ appear only in $A_{*}^{\prime}$; hence $R^{\prime}+A_{s}^{\prime}$ is semireduced. By Case 1 , we have $e\left(G^{\prime}\right) \geq e_{n}$, provided only that $n^{\prime}=v\left(G^{\prime}\right)$ is sufficiently large. Thus if we can select $Q$ so that $n=n^{\prime}$ and $e(G) \geq e\left(G^{\prime}\right)$, the desired result follows. We shall see that such a selection is possible provided $v(C)$ is sufficiently large. In the remaining case, i.e. when $v(C)$ is bounded by an appropriate constant, we make a different selection of $Q$ which does not yield $n^{\prime}=n$. Nevertheless, the boundedness of $v(C)$ guarantees that $n^{\prime}$ is large whenever $n$ is sufficiently large, and we are still able to obtain $e(G) \geq e_{n}$. The details are presented below.

Symbolically we may write $G-G^{\prime}=C-D+A_{*}-Q$. If we let $k=v(C)-$ $v(D)+v\left(A_{*}\right)$ then $n-n^{\prime}=k-v(Q)$. If $v(C)$ is sufficiently large, $k \geq v\left(A_{*}\right)$ and $Q_{k}(\mathfrak{I})$ exists. Taking $Q=Q_{k}(\mathfrak{I})$ gives $n^{\prime}=n$ and $e(G) \geq e\left(G^{\prime}\right)$ as desired. (Since here $e(G)-e\left(G^{\prime}\right)=d(G)-d\left(G^{\prime}\right)=r(C)+d\left(A_{*}\right)-d(Q)$ and, via Lemma $1, d\left(A_{*}\right) \geq d(Q)$.) It is not difficult to obtain a crude bound $K$ so that $v(C) \geq K$ guarantees the appropriate conditions on $k$. E.g. $v(C)-v(D)$ $\geq N_{0}(2 s)$ ensures the existence of $Q_{k}(\mathfrak{T})$. If we let

$$
L=v\left(\mathfrak{I}_{s}\right) \cdot \sum_{1 \leq i \leq s}\left(m_{i}-1\right)
$$

then certainly $v(D) \leq L$; hence we can take $K=L+N_{0}(2 s)$.

Now we turn to the case in which $v(C)<K$. Here we select $Q=A_{*}$, so that $G^{\prime}=G-C+D$. Consider the bracketed terms in the decomposition of 
$e(G)-e_{n}$ as $\left\{e(G)-e\left(G^{\prime}\right)\right\}+\left\{e\left(G^{\prime}\right)-e_{n^{\prime}}\right\}+\left\{e_{n^{\prime}}-e_{n}\right\}$. Since $c\left(G^{\prime}\right)=$ $c(G)$, we have $e(G)-e\left(G^{\prime}\right)=n-n^{\prime}+r(C)$. Also, $e_{n^{\prime}}-e_{n}=n^{\prime}-n+z$ where $z=d\left(Q_{m^{\prime}, s}\right)-d\left(Q_{m, s}\right)$ and $m^{\prime}=n^{\prime}-v_{0}$. Thus $e(G)-e_{n}=\left\{e\left(G^{\prime}\right)-\right.$ $\left.e_{n^{\prime}}\right\}+r(C)+z$. Since $n-n^{\prime}=v(C)-v(D)$, the assumption that $v(C)<K$ implies that $n^{\prime}>n-K$. This guarantees that for $n$ sufficiently large we have $e\left(G^{\prime}\right) \geq e_{n^{\prime}}$. (E.g. this is assured if $n \geq K+v_{0}+N_{v_{0}, s}$ and in what follows we assume $n$ is at least this large.)

So we need only show that $r(C)+z \geq 0$ provided $n$ is big enough. If $v(C) \geq v(D)$ then $n \geq n^{\prime}$, hence $z \geq 0$ via Lemma 1. Since always $r(C) \geq 0$, there is nothing further to prove here. Thus suppose $v(C)<v(D)$. We will show that this implies $r(C)>0$, hence require only $z \geq-1$. By Lemma 2, this last condition is met whenever $n$ is sufficiently large (e.g. whenever $n \geq v_{0}+N_{l, s}$ for any integer $l \geq v(D)-v(C)$; in particular we may take $l=$ $L$ ). To see that $r(C)>0$, simply observe that $v\left(C_{i}\right) \geq v\left(D_{i}\right)$ whenever $C_{i}$ is a tree (since $D_{i}$ lies in $\mathfrak{T}_{s}$ but $C_{i}$ does not). Thus the assumption that $v(C)<v(D)$ implies that $C$ cannot be a forest.

Thus in all cases, $e(G) \geq e_{n}$ whenever $n$ is sufficiently large. In fact, we have shown that this holds whenever $n \geq v_{0}+L+N$ where $N$ is the maximum of $N_{0}(2 s), N_{v_{0}, s}$ and $N_{L, s}$.

5. Consequences of the main result. It follows from the theorem just proved that for an arbitrary group $\varrho$, as $n$ grows large $e(\varrho, n)$ grows at the same rate as $e(i d, n)$. Indeed, for all $n$ sufficiently large we have

$$
d_{0}+s \leq e(\mathcal{G}, n)-e(i d, n) \leq d_{0}+s+1 .
$$

To see this, use the fact that for $n$ large enough

$$
e(\text { id, } n)=e\left(Q_{n}\right)=n-c\left(Q_{n}\right)
$$

and $e(\varrho, n)$ is given by (4.1) with $m=n-v_{0}$ and $s=s(\varrho)$. Observe also that if $u_{s}=v\left(T_{1}+T_{2}+\ldots+T_{s}\right)$ and $n^{\prime}=u_{s}+m$, then $Q_{n^{\prime}}=T_{1}+T_{2}+$ $\ldots+T_{s}+Q_{m, s}$, hence

$$
e(\Theta, n)=n+d_{0}+s-c\left(Q_{n^{\prime}}\right) .
$$

Thus $e(乌, n)-e(i d, n)=d_{0}+s+x$ where $x=c\left(Q_{n}\right)-c\left(Q_{n^{\prime}}\right)$. It is easy to see that $v_{0} \geq u_{s}$, so that $n \geq n^{\prime}$ and an application of Lemma 2 yields $0 \leq x \leq 1$ as desired.

In view of the above, the rate of growth of $c\left(Q_{n}\right)$ may be of some interest, as is the problem of reasonably explicit computation of these numbers. In this regard, we remark only that $c\left(Q_{n}\right)$ can be described as in [13] in terms 
of $a_{k}$, the number of nonisomorphic asymmetric trees on $k$ vertices. Let $A_{j}=1 a_{1}+2 a_{2}+\ldots+j a_{j}$ and let $u$ be maximal subject to $A_{u} \leq n$. Then $c\left(Q_{n}\right)=a_{1}+a_{2}+\ldots+a_{u}+w$, where $w$ is the greatest integer in $\left(n-A_{u}\right) /(u+1)$. Although no closed formula for $a_{k}$ is known, the generating function for this sequence is obtained in [8].

Leaving aside the problem of actual computation of $c\left(Q_{n}\right)$, in view of our stability theorem the complete determination of $e(\oiiint, n)$ for all $n$ can be separated into several distinct subproblems:

(i) determine the values of $s\left(\biguplus_{)}, d_{0}\right.$ and $v_{0}$;

(ii) determine the point at which stability first occurs; i.e. find the smallest integer $N_{0}$ such that $e(\varrho, n)=e_{n}$ for $n \geq N_{0}$;

(iii) investigate the behavior of $e(\varrho, n)$ when $n<N_{0}$.

It is hoped that this reformulation may be helpful in future work on the minimum edge problem. The stability result obtained here has little direct bearing on (ii) and (iii), although it is possible that in particular instances some of the ideas used in the proof may be of assistance. With regard to (i), computation of $s\left(\biguplus_{)}\right)$is a purely algebraic problem, of course, but determination of $d_{0}$ and $v_{0}$ may involve both algebraic and graph theoretic techniques. Also, (i) is closely related to the problem of actually exhibiting a "stability

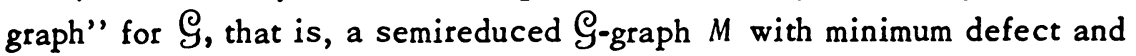
which attains the minimum number of vertices among such graphs. We offer several observations on the structure of $M$.

While the stability graph for a given group $\mathcal{G}$ is by no means uniquely determined, some uniformizing assumptions can be made. In order to attain the minimum defect $d_{0}$ among semireduced $\mathcal{S}_{\text {-graphs, }} M$ must contain precisely $s=s\left(\biguplus_{\zeta}\right)$ nonisomorphic asymmetric components. Since we also require $M$ to have the minimum number $v_{0}$ of vertices, we may take these components to be the trees $T_{1}, T_{2}, \ldots, T_{s}$ and if $m_{i}$ denotes the multiplicity of $T_{i}$ in $M$ we can also assume that $m_{i} \geq m_{i+1}$ for $1 \leq i<s$.

If $M$ is reduced (i.e. if $m_{s}>1$ ), the $m_{i}$ are just the degrees of the nontrivial symmetric direct factors of $\mathcal{G}$, and we have

$$
M=m_{1} T_{1}+m_{2} T_{2}+\cdots+m_{s} T_{s}+M_{0}
$$

where $M_{0}$ is a reduced $\varrho_{0}$-graph. Here $\varrho_{0}$ is a direct factor of $\varrho_{\text {which }}$ is maximal subject to $s\left(\varrho_{0}\right)=0$. It is clear that $M_{0}$ is a stability graph for $\varrho_{0}$, and it follows that $M_{0}$ has no asymmetric components.

If the stability graph $M$ always turned out to be a reduced $\mathcal{C}_{\text {-graph, we }}$ could confine attention to groups with no nontrivial symmetric direct factors, for in view of the above observation, the general situation would present no 
additional difficulties. Unfortunately, this is not the case.

For example, if $\mathcal{S}_{2}$ appears as a direct factor of $\mathcal{G}$, then the stability graph $M$ is definitely not reduced whenever $s(\zeta)>1$. For suppose $M$ were reduced. We assume that for $i \leq s, T_{i}$ has multiplicity $m_{i}>1$ in $M$, and $m_{s}=2$. Consider the graph $M_{1}$ obtained from $M$ by replacing one copy of $T_{s}$ by a path $P_{2}$ of length 1 . Then $M_{1}$ is a semireduced $\Theta_{\text {-graph having }}$ the same defect as $M$ but with fewer vertices. This contradicts the minimality of $v_{0}$.

We remark that although $M$ itself need not be reduced, there always exists a reduced $\varrho_{\text {-graph }} R$ so that $e(\varrho, n)$ is attained for large $n$ by $R+$ $Q_{n^{\prime}, t}$ where $n^{\prime}=n-v(R)$ and $t=a(R)$. (To see this, just take $R$ to be the reduced part of the stability graph $M$.) This fact would be much more interesting if we could give a direct characterization of $R$, or at least of $v(R)$ and possibly $a(R)$. In some instances, $R$ may be described in exactly the same manner as $M$. More precisely, let $d_{1}$ be the minimum defect of all reduced 9 -graphs, and among such graphs attaining $d_{1}$ let $R_{1}$ be one having

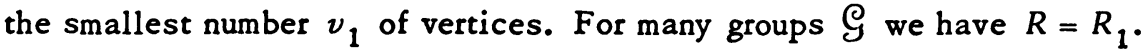
If this were always the case, the emphasis on semireduced graphs in the theorem would be misplaced. We give an example in which this is not so.

Let $\mathcal{G}=\delta_{3} \times S_{2}$. We have $s(\mathcal{G})=2$ and it is easy to see that $d_{1}=-5$, $v_{1}=17$ and $R_{1}=3 T_{1}+2 T_{2}$. However, $d_{0}=-5, v_{0}=12$ and $M=3 T_{1}+$ $P_{2}+T_{2}$ where $P_{2}$ is a path having two vertices. Thus $R=3 T_{1}+P_{2} \neq R_{1}$. More important, for infinitely many $n, e\left(\biguplus_{,} n\right)$ is not attained by $R_{1}+$ $Q_{n-17,2}$. For, letting $m=n-12$ and $m^{\prime}=n-17$ we have $c\left(Q_{m, 2}\right)>$ $c\left(Q_{m^{\prime}, 2}\right)$ and hence $e\left(M+Q_{m, 2}\right)<e\left(R_{1}+Q_{m^{\prime}, 2}\right)$ whenever $n=v\left(M+F_{t}\right)$ where $T_{1}+T_{2}+F_{t}$ is the sum of the first $t$ trees in the standard list.

We make one final observation. There always exists a graph $M^{\prime}$ such that for $n$ sufficiently large, $e(\varrho, n)$ is attained by $M^{\prime}+Q_{n^{\prime}}$ where $n^{\prime}=$ $n-v_{0}+u_{s}$ as in (5.3). To see this, simply select $M$ so that $T_{i}$ has multiplicity $>0$ in $M$ for $1 \leq i \leq s$. If $M=M^{\prime}+T_{1}+\ldots+T_{s}$ then $M+Q_{m, s}=$ $M^{\prime}+Q_{n^{\prime}}$. Of course, $M^{\prime}$ will not generally be a $\mathcal{G}_{\text {-graph. }}$

6. Application to the commutative case. A good general description can be given for the stability graph of an arbitrary commutative group. Assume in what follows that $\mathcal{G}$ is commutative. Let $s=s(\mathcal{G})$ and let $\mathcal{G}_{0}$ denote a maximal direct factor of $\varrho_{\text {, with }} s\left(\bigodot_{j_{0}}\right)=0$. When $s=0, \mathcal{G}=\mathcal{G}_{0}$. When $s>0$, $\mathcal{G}=\Theta_{s} \times \Theta_{0}$ where $\Theta_{s}$ is the direct product of $s$ copies of a cyclic group of order 2 .

We will employ the following result whose proof is omitted. 
Lemma 3. Let $C$ be a component of multiplicity $m$ in some S-graph, $^{-}$ where $\mathrm{G}$ is commutative.

(1) If $m>1$, then $m=2, C$ is asymmetric, and $\mathcal{G}$ has a direct factor of order 2 arising from $C$.

(2) If $C$ is a tree, then $\mathrm{P}_{u t}(C)$ is either trivial or a direct product of cyclic groups of order 2.

(3) If $C$ is unicyclic and (Put $(C)$ has no direct factors of order 2, then Qut $(C)$ is cyclic.

Suppose now that $H$ is a semireduced $\varrho_{-g r a p h}$. It follows immediately from (1) and (2) that $d(H) \geq-2 s$. Moreover, if equality holds here then a good deal can be said regarding the structure of $H$. Assume $d(H)=-2 s$ and $s>0$. We can write $H=H_{s}+H_{0}$ where $H_{s}$ has precisely $2 s$ components each of which is a tree whose automorphism group has order $\leq 2$, and each component of $H_{0}$ has defect 0 , i.e. is unicyclic. Furthermore, to obtain $c\left(H_{s}\right)=2 s$ it is necessary that $a\left(H_{s}\right)=s$ and $C_{u t}\left(H_{s}\right)$ has order $2^{s}$. It follows that $H_{s}$ is a semireduced $\varrho_{s}$-graph and $H_{0}$ a reduced $\varrho_{0}$-graph. Also, if $C$ is a component of $H_{0}$ then $C$ has multiplicity 1 , is unicyclic and Qut $(C)$ is cyclic of order $>2$. When $s=0, H_{0}=H$ and has the same structural properties as when $s>0$.

Further insight into the structure of the components of $H_{0}$ is provided by the following observation. Let $U$ be an asymmetric unicyclic graph, and $q>2$. Let $U(q)$ denote the unicyclic graph on $q \cdot v(U)$ vertices obtained by taking the natural $q$-fold covering of $U$. (If we regard $U$ as a necklace, then $U(q)$ is obtained by unclasping $U$, taking $q$ copies of this unclasped necklace and joining their clasps in the most natural manner.) It can be shown that $\mathfrak{C u t}_{u}(U(q)) \cong \mathcal{C}_{q}$, the cyclic group of order $q$, and that every unicyclic $C_{q}$-graph arises in this manner. A detailed proof will be provided elsewhere.

Finally, we show that $d_{0}=-2 s$ by exhibiting a semireduced $\varrho_{-g r a p h}$ $H$ with $d(H)=-2 s$. Take $H=2\left(T_{1}+T_{2}+\ldots+T_{s}\right)+H_{0}$ where $H_{0}$ is as follows. Decompose $\mathcal{G}_{0}$ as a direct product of cyclic groups in some manner, and let the orders of the factors in this decomposition be $q_{1}, q_{2}, \ldots$, $q_{k}$. Let $U_{1}, U_{2}, \ldots, U_{k}$ be asymmetric unicyclic graphs chosen so that whenever $q_{i}=q_{j}$ with $i \neq j, U_{i}$ is not isomorphic to $U_{j}$. Take $H_{0}$ to be the sum of the graphs $U_{i}\left(q_{i}\right)$ for $1 \leq i \leq k$. Each component of $H_{0}$ has multiplicity 1 , hence $H_{0}$ is a $\varrho_{0}$-graph. It is clear that $H$ meets the desired conditions.

We can obtain a stability graph $M$ for $\varrho$ by modifying the above construction 
to attain a minimum number of vertices. In more detail, it is clear from the result on the structure of semireduced $\xi_{\text {-graphs }}$ of minimum defect that it is sufficient to obtain stability graphs $M_{0}, M_{s}$ for $\Theta_{0}, \varrho_{s}$ respectively. Setting $M=M_{0}$ when $s=0$, and $M=M_{s}+M_{0}$ when $s>0$ yields a stability graph $M$ for ${ }_{\text {. }}$.

When $s>0, M_{s}$ is obtained as follows. Consider all nonisomorphic trees having automorphism group of order $\leq 2$, and list them in order of increasing number of vertices: $W_{1}, W_{2}, \ldots$ Take

$$
M_{s}=T_{1}+\cdots+T_{s}+W_{1}+\cdots+W_{s} ;
$$

clearly $M_{s}$ is a stability graph for $\varrho_{s}$.

It is easy to describe an explicit procedure for obtaining $M_{0}$, but we gloss over the details. The procedure can be summarized as follows. To each decomposition $D$ of $\mathcal{G}_{0}$ as a direct product of cyclic groups we assign a standard minimum-vertex $\Theta_{0}$-graph $M_{D}$ whose components are unicyclic and have the factors appearing in $D$ as their automorphism groups. If $D$ is such that $v\left(M_{D}\right)$ is a minimum, we take $M_{0}=M_{D}$.

The explicit description of $M_{D}$ has been omitted here, but is not hard to reconstruct. The numbers $v\left(M_{D}\right)$ can be described entirely in terms of the orders of the factors in the decomposition $D$ and the sequence $b_{k}$. Here $b_{k}$ denotes the number of nonisomorphic asymmetric unicyclic graphs on $k$ vertices; the generating function for $b_{k}$ is known [15]. There seem to be genuine subtleties involved, however, in deciding which decomposition $D$ minimizes $v\left(M_{D}\right)$. For a specific group $\varrho_{0}$, this can be settled by straightforward computation, of course, but the outcome in the general case cannot readily be described in simple terms as yet. There is, however, an important special case in which the result is clear.

Theorem. Let $q_{1}, q_{2}, \ldots, q_{k}$ denote the orders of the factors in the decomposition of $\mathcal{G}_{0}$ as a direct product of cyclic groups of prime-power order. If $q_{i} \neq q_{j} \neq 2$ for $i \neq j(1 \leq i, j \leq k)$, then $M_{0}=C_{1}+C_{2}+\ldots+C_{k}$ where $C_{i}=U\left(q_{i}\right)$ and $U$ is the unicyclic asymmetric graph on 6 vertices. In particular, $v\left(M_{0}\right)=6\left(q_{1}+q_{2}+\ldots+q_{k}\right)$.

As an immediate corollary, we obtain the following explicit description of the stability graph $M$ in the case when $\mathcal{G}$ is cyclic. Suppose $\mathcal{G}$ is cyclic of order $q=q_{1} q_{2} \ldots q_{k}$ where the $q_{i}$ are powers of different primes. If $q \neq 2 \bmod 4$, then $s(\varrho)=0$, hence $M=M_{0}$ as in the theorem. In particular, $d_{0}=0$ and $v_{0}=6 \bar{q}$ where $\bar{q}=q_{1}+q_{2}+\ldots+q_{k}$. If $q \equiv 2 \bmod 4$, then $s\left(\mathcal{G}_{)}=1\right.$, hence $\mathcal{G}_{0}$ is cyclic of order $q / 2$. We have $M=2 T_{1}+M_{0}$ where 
$M_{0}$ is the stability graph for $\varrho_{0}$, as above. In particular, $d_{0}=-2$ and $v_{0}=$ $2+6(\bar{q}-2)$.

\section{REFERENCES}

1. R. Frucht, Herstellung von Graphen mit vorgegebener abstrakter Gruppe, Compositio Math. 6 (1938), 239-250.

2. - On the groups of repeated graphs, Bull. Amer. Math. Soc. 55 (1949), 418-420. MR 10, 615.

3. R. Frucht, A. Gewirtz and L. V. Quintas, The least number of edges for graphs having automorphism group of order three, Recent Trends in Graph Theory (Proc. Conf., New York, 1970), Lecture Notes in Math., vol. 186, Springer-Verlag, Berlin and New York, 1971, pp. 95-104. MR 43 \#6119.

4. A. Gewirtz, A. Hill and L. V. Quintas, Extremum problems concerning graphs and their groups, Combinatorial Structures and Their Applications (Proc. Calgary Internat. Conf. Combinatorial Structures and Their Applications, Calgary, Alberta, 1969), Gordon and Breach, New York, 1970, pp. 103-109. MR 42 \# 108.

5. G. Haggard, The least number of edges for graphs having dihedral automorphism group, Discrete Math. 6 (1973), 53-78. MR 48 \# 157.

6. M. Hall, Jr., The theory of groups, Macmillan, New York, 1959. MR 21 \#1996. \#1566.

7. F. Harary, Graph theory, Addison-Wesley, Reading, Mass., 1969. MR 41

8. F. Harary and G. Prins, The number of homeomorphically irreducible trees, and other species, Acta Math. 101 (1959), 141-162. MR 21 \#653.

9. A. Kerber, Representations of permutation groups. I, Lecture Notes in Math., vol. 240, Springer-Verlag, Berlin and New York, 1971.

10. B. H. Neumann, Lectures on topics in the theory of infinite groups, Tata Institute of Fundamental Research, Bombay, 1960.

11. O. Ore, The theory of graphs, Amer. Math. Soc. Colloq. Publ., vol. 38, Amer. Math. Soc., Providence, R. I., 1962. MR 27 \# 740.

12. - Theory of monomial groups, Trans. Amer. Math. Soc. 51 (1942), 15-64. MR 3, 197.

13. L. V. Quintas, Extrema conceining asymmetric graphs, J. Combinatorial Theory 3 (1967), 57-82. MR 35 \#2780.

14. - The least number of edges for graphs having symmetric automorphism group, J. Combinatorial Theory 5 (1968), 115-125. MR 37 \# 6211.

15. P. K. Stockmeyer, The enumeration of groups with prescribed automorphism group, Ph. D. Dissertation, University of Michigan, Ann Arbor, Mich., 1971.

16. G. Haggard, D. McCarthy and A. Wohlgemuth, Extremal edge problems for graphs with given hyperoctahedral automorphism group (to appear).

DEPARTMENT OF MATHEMATICS, ST. JOHN'S UNIVERSITY, JAMAICA, NEW YORK 11439

DEPARTMENT OF MATHEMATICS, PACE UNIVERSITY, NEW YORK, NEW YORK 10038 